Guillaume Robin, Berghain, Techno und Körperfabrik. Ethnographie eines Stammpublikums.

Jean-Louis Georget

\title{
OpenEdition
}

Journals

Édition électronique

URL : https://journals.openedition.org/ifha/11544

DOI : 10.4000/ifha. 11544

ISSN : 2198-8943

Éditeur

IFRA - Institut franco-allemand (sciences historiques et sociales)

Référence électronique

Jean-Louis Georget, « Guillaume Robin, Berghain, Techno und Körperfabrik. Ethnographie eines

Stammpublikums. », Revue de l'IFHA [En ligne], Date de recension, mis en ligne le 01 novembre 2021

consulté le 03 novembre 2021. URL : http://journals.openedition.org/ifha/11544 ; DOI : https://doi.org/ 10.4000/ifha. 11544

Ce document a été généré automatiquement le 3 novembre 2021

(C)IFHA 


\section{Guillaume Robin, Berghain, Techno und Körperfabrik. Ethnographie eines Stammpublikums.}

Jean-Louis Georget

\section{RÉFÉRENCE}

Guillaume Robin, Berghain, Techno und Körperfabrik. Ethnographie eines Stammpublikums, Büchner, 2021, 176 p. 
Le Berghain vient de rouvrir. Il fait partie de la mythologie berlinoise et de l'image de l'underground qui a façonné au fil des années le paysage urbain, puis s'est transformé insidieusement en scène officielle, supervisée par la capitale allemande qui en tirait, avant la crise sanitaire, 1,8 milliards de revenus par an. Cet univers reste néanmoins secret aux non-initiés, notamment son bateau-amiral le Berghain, discothèque connue dans le monde entier, qui tire sa réputation à la fois du tri arbitraire de sa clientèle à l'entrée et $\mathrm{du}$ culte qui l'entoure. L'ouvrage de Guillaume Robin, fruit d'une enquête menée sur plusieurs années, vient décrypter pour un large public dans une langue parfaitement accessible les codes de cet univers qui s'inscrit profondément dans l'espace urbain.

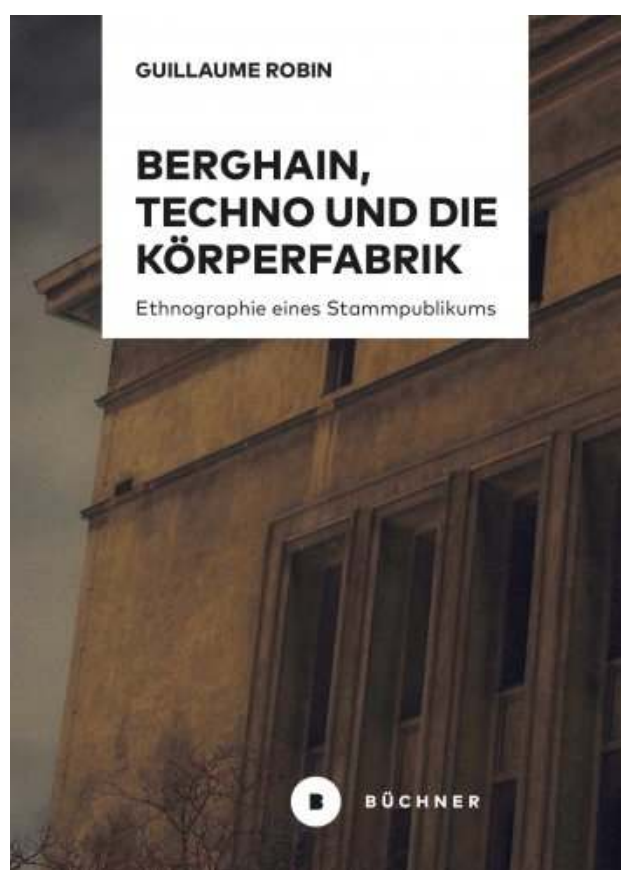

Contrairement à l'image de détente qu'elle souhaite diffuser, l'organisation du Berghain obéit à des règles drastiques pour dispenser l'oubli. La séquence est quotidienne, commençant le samedi soir et s'achevant le lundi matin, dans un espace hétérotopique qui permet la libération des corps et leur expression dans la transe et les sexualités multiples. Si son nom est l'acronyme de la jonction de deux quartiers, l'un à l'Ouest, l'autre à l'Est, c'est bien le caractère oriental de cette ancienne usine électrique désaffectée qui lui donne sa physionomie. Dans cette ancienne zone démilitarisée et peu construite se sont installés dans les années 1990 nombre d'établissements et s'est développée une contre-culture des techno-partys hédonistes, où se mêlent tous les expériences, qui s'appuient également sur une consommation de drogues variées comme le montre l'auteur. On se voue au plaisir éphémère et intemporel, puisqu'aucune horloge ne mesure la durée de la fusion dans l'événement collectif, l'auteur osant avec une certaine réussite la comparaison avec le culte des mystères de l'Antiquité.

Ce culte est régi par des règles qui, si elles sont ignorées ou transgressées, ferment les portes des impétrants imprudents. Elles sont d'autant mieux respectées qu'elles sont intériorisées, ces " bacchanales » étant une boîte noire et opaque où toute diffusion des images sur les réseaux sociaux aurait un effet de désacralisation des lieux.

Si la plupart des ouvrages s'intéressent aux acteurs de la scène techno, le rédacteur de l'étude entre par la médiation des acteurs et de leurs corps dans l'espace consacré. Les surprises sont nombreuses tant Berlin se distingue du Paris des années 1980 ou du Londres des années 1990 : le public étudié ne correspond pas à l'idée que l'on se fait de la musique techno, puisque la moyenne d'âge oscille plutôt entre 30 et 40 ans, l'anecdote du garçon y passant la soirée avec sa mère et son amie $\mathrm{y}$ étant particulièrement croustillante. Mais ces adeptes de la fête récurrente ont également un profil étonnamment varié, la liste des nations représentées étant particulièrement étoffée, depuis l'Europe de l'Ouest jusqu'au Kirghizstan. En outre, ce public est à la fois 
sédentaire et berlinois, mais aussi très mobile puisqu'une grande partie de l'échantillon fait plusieurs fois par an le trajet depuis des destinations variées (jusqu'à Dubaï!) pour venir se plonger dans les nuits berlinoises.

Le livre se compose de quatre grands chapitres et d'une conclusion. Le premier, qui s'intitule «Les corps dans l'espace » livre une savante géographie du labyrinthe que constitue la mythique discothèque, depuis son entrée sous le regard surfait et patibulaire de Sven Maquardt, le cerbère des lieux. Guillaume Robin montre bien comment le système fonctionne selon le principe ritualisé de l'inclusion et l'exclusion. Le monde des plaisirs est régulé par un pouvoir disciplinaire fort, qui signifie pas à pas la marche à suivre: tenue vestimentaire, attente, orientation de l'éclairage, passage successif des seuils liminaires jusqu'à l'acceptation et l'intégration par la communauté. Cette inclusion est une entrée dans un monde en apparence merveilleux où s'abolissent par là-même les frontières sociales, du moins pour la durée où les corps peuvent tenir le rythme, souvent pendant une dizaine d'années avant l'usure pérmaturée. Cette communauté sait s'organiser, par exemple comme cela s'est produit en 2019 contre la hausse des prix, mais sans effet sur la direction de l'établissement, qui reste absolument hermétique à toutes les injonctions qui pourraient remettre en cause l'ordre établi. Surveiller et punir, tel est le prix de cette liberté. Comme le souligne l'auteur, le personnel de surveillance est partout présent, stratégiquement placé, la dénonciation bienvenue. Il y a même un système d'archivage pour les bannis, notamment les vendeurs de produits illicites que par ailleurs tout le monde consomme. Tout doit rester dans l'ombre de la législation tacite qu'impose le Berghain, parfois de manière parfaitement arbitraire. L'exclusion du Berghain s'accompagne de toutes sortes d'humiliations, parfois violentes. Elle signifie une petite mort, un déni de corps dont les stigmates sont parfois consignés, à l'aide de tatouages par exemple, comme la marque de combats passés.

Dans le second chapitre intitulé «Les corps utopiques", l'auteur décrit avec minutie le processus de délivrance du corps dont on ne peut s'échapper. Cette anonymisation se produit par une disparition de la personne qui n'a aucun moyen de se refléter sinon dans le regard des autres, tout miroir ou surface permettant de s'entrevoir étant absente du lieu. Ce corps qui se fonde dans la masse tente toutefois de s'en extirper par son accoutrement, dont l'aspect est parfois presque carnavalesque, depuis le tablier de brasseur jusqu'au voile de nonne pour être reconnue de la communauté dans la fusion imposée. Mais cet uniforme, qui fait de la scène une Commedia delle Arte, résulte d'un jeu de rôle, qui fige pour un temps la communauté établie. L'antre berlinois qui fait disparaitre les formes d'auto-désignation est un lieu de perdition individuel qui appelle une réassurance collective permanente.

Car le corps se livre pendant 16 heures tout entier à la danse, c'est-à-dire l'équivalent de deux marathons. La musique est bien la première motivation des participants. Même si la techno n'impose pas de figures de style reconnues à l'instar d'autres pratiques chorégraphiques, un style se dégage néanmoins. Ce qui le caractérise est avant tout son énergie, même si le périmètre personnel de chacun est respecté dans une forme d'individualisme de bon aloi. La musique, comme le dit le rédacteur du livre, est composée de loops et samples qui peut aller jusqu'à 140 beats par minute. La danse devient transe et l'élément fort de cette socialisation qui unit tous les acteurs. Néanmoins, les corps, soumis à toutes sortes d'emprises artificielles, restent sous contrôle et la plupart des participants savent comment maîtriser les nuits 
interminables, à coups de litres d'eau, de nourriture appropriée et de pratiques sportives drastiques.

Le troisième chapitre est consacré à « La robotisation de la piste de danse ». Partout, la confrontation binaire entre les genres est remise en question. Le territoire de la discothèque est l'objet de la déconstruction des sexualités. L'histoire du club suit également celle de la dépénalisation progressive de l'homosexualité depuis les années 1970. Le chemin qui mène au Berghain est souvent un parcours parsemé de résidus de préjugés contre les minorités, même dans une ville aussi ouverte que Berlin. Mais une fois à l'intérieur, le lieu consacré constitue un havre de paix pour la diversité sexuelle, dans lequel règne une certaine concorde, même si le concept d'espace sûr mérite réflexion, dans la mesure où il n'est sûr que pour un certain type de public en fonction de l'orientation de la soirée choisie. L'ambiguïté est la norme, le modèle hétérosexuel n'est nullement dominant et le mélange des genres en constitue même par certains côtés un détournement. Guillaume Robin dessine une typologie habile et différenciée, où se mêlent les expériences de vie les plus diverses et où la piste devient le lieu de tous les fantasmes et scènes imaginables. Ce brouillage des sexes est exigé par le public luimême, selon les deux modalités que sont d'un côté le trouble qu'occasionnent les mélanges et son corollaire qu'est la robotisation des corps. Il n'y a plus dans la nuit intempestive que des danseurs ou des danseuses qui confondent leurs attributs et subissent sous la pression du lieu une confusion temporaire des genres. Y sont également naturellement présents les icônes du Berlin depuis les années 1920 que sont les travestis.

Le livre, dans un dernier chapitre, s'arrête sur les conséquences de la crise sanitaire pour les établissements berlinois. Il décrit toute la vitalité de l'économie qui tourne autour des 9000 personnes employées dans les clubs et signifie que la crise a été le révélateur de l'ancrage profond des pratiques évoquées dans la culture berlinoise. En effet, au fur et à mesure que les clubs connaissaient des difficultés, se créaient des associations et des solidarités pour reconstituer artificiellement la communauté des adeptes des nuits berlinoises. Il y a un beau passage sur les fêtes clandestines et l'émergence de nouveaux lieux dans les bâtiments abandonnés, Berlin ayant peur du vide et chaque interstice, bâtiment vide, forêt ayant été finalement un appel à faire revivre furtivement ce pétillement berlinois.

Le livre se termine avec le chapitre "Closing ", très explicite, qui a lieu en général le lundi matin, où le public éprouve un sentiment oscillant entre l'impuissance et le nirvana. On espère une suite, à la manière du public, à cette étude ethnographique très réussie pour continuer d'explorer la vitalité de la nuit berlinoise.

\section{AUTEUR}

JEAN-LOUIS GEORGET

Université Sorbonne Nouvelle 